\title{
Experimentally induced central sensitization evokes segmental autonomic responses in humans
}

\begin{abstract}
Objective: Central sensitization has been associated with the pathophysiology of somatoautonomic responses however, the causal role of central sensitization in the clinical manifestation of somatoautonomic responses has not previously been demonstrated in humans. The purpose of this study was to test the hypothesis that a temporal relationship exists between experimentally induced central sensitization and skin temperature change within neurosegmentally linked dermatomes in young healthy humans.
\end{abstract}

Methods: A total of 15 test (8 males, 7 females; age 21.2(1.3) years) and 12 control (5 males, 7 females; age 21.2(1.3) years) subjects were assessed. Central sensitization was induced using topical capsaicin (Zostrix, 0.075\%), targeting the C3-C5 spinal levels in test subjects; controls received a non-sensitizing placebo cream. Skin temperature changes in segmentally linked versus segmentally distinct dermatomes were compared between groups at baseline, 10,20 and 30 minutes post-sensitization, using infrared thermography.

Results: Significantly greater decreases in average skin temperature were observed in the segmentally-linked dermatomes of test subjects versus controls at all time intervals $(10,20,30$ minutes $)$ post-sensitization $(\mathrm{p}<0.001)$. Significantly decreased average skin temperatures were also observed in the segmentally linked dermatomes versus segmentally distinct dermatomes of test subjects, at all time intervals $(\mathrm{P}<0.050)$; these differences were not observed in controls.

Conclusion: Our findings demonstrate neurosegmentally distributed skin temperature changes after experimental induction of central sensitization in healthy humans. These are the first observations in humans that offer support to the theory that central sensitization may be a contributing physiologic mechanism in the clinical expression of somatoautonomic responses in humans.

Keywords: central Sensitization, somatovisceral, thermography, autonomic, functional gastrointestinal disorder
Volume I Issue 3 - 2017

\author{
John Z Srbely, ' James P Dickey, ${ }^{2}$ Yuri R \\ Montaholi ${ }^{3}$ \\ 'Department of Human Health and Nutritional Science, \\ University of Guelph, Canada \\ ${ }^{2}$ School of Kinesiology, Western University, Canada \\ ${ }^{3}$ Department of Animal Science \& Aquaculture, Dalhousie \\ University, Canada
}

Correspondence: John Z Srbely, Department of Human Health and Nutritional Science, University of Guelph, Canada, Tel 519-824-4I20, Fax 519-763-5902, Email jsrbely@uoguelph.ca

Received: April 25, 2017| Published: June 20, 2017
Abbreviations: ANS, autonomic nervous system; T10,10 minutes post-capsaicin; T20, 20 minutes post-capsaicin; T30, 30 minutes post-Capsaicin; SMT, spinal manipulative therapy

\section{Introduction}

The interaction of the somatosensory and autonomic pathways is important to normal function and adaptation of an organism to its environment. Research in animals and humans shows that the somatosensory and autonomic pathways interact at all levels of the nervous system. ${ }^{1}$ Somatosensory input from both cutaneous and deep tissues has demonstrated the capacity to modulate the output of the autonomic nervous system (ANS), ${ }^{2}$ this phenomenon is known as the somatoautonomic reflex and has been confirmed in both animals ${ }^{3}$ and humans. ${ }^{4}$ Despite the accumulating body of literature demonstrating the significant impact of somatosensory input on autonomic regulation, the underlying physiologic mechanisms of this phenomenon are still poorly understood. ${ }^{5}$

Research with animals ${ }^{2}$ and humans ${ }^{6,7}$ demonstrates that noxious input from both cutaneous and deep somatic tissues evokes systematic autonomic responses. Animal models demonstrate that pain arising from incision of the hindpaw leads to persistent colonic hypersensitivity. ${ }^{8}$
Furthermore, noxious movements of the knee joint in animals increase both heart rate and blood pressure, ${ }^{9}$ analogous sympathetic responses are not observed during innocuous movements of the knee. Moreover, research in animals suggests that these somatoautonomic responses may manifest in neurosegmental patterns, ${ }^{10-12}$ however the precise physiologic mechanism is still unclear. The mechanism responsible for cross-sensitization of somatic and visceral tissues may be linked to the phenomenon of central sensitization, characterized as hyperexcitability of neurons within the central nervous system. ${ }^{13}$ Central sensitization has been previously linked to the development and maintenance of visceral hypersensitivity ${ }^{14}$ and viscerosomatic pain referral in animals. ${ }^{15}$ Similarly, we theorize that central sensitization may play a causal role in the clinical manifestation of somatoautonomic responses in humans.

The overall purpose of this line of inquiry is to explore the causal role of central sensitization in the clinical manifestation of somatoautonomic response in humans. In this foundational study, we aimed to investigate the temporal relationship between experimentally induced central sensitization and neurosegmentally distributed changes in skin temperature. We tested the hypothesis that experimentally increasing central sensitization within a target spinal segment leads to systematic changes (decreases) in skin temperature within 
neurosegmentally linked dermatomes in healthy humans. Evidence of a neurosegmental response would provide additional evidence to the theory supporting the causal role of central sensitization in the pathophysiology and clinical manifestation of somatoautonomic responses in humans. Much of the empirical evidence in this field is derived from animal models ${ }^{2}$ and, to date, we are unaware of any group investigating the temporal association between central sensitization and neurosegmentally mediated skin temperature change in humans using an experimental in vivo placebo controlled design.

\section{Materials and methods}

\section{Data collection}

This study was approved by the Ethics Committee and was carried out in accordance with the Declaration of Helsinki (2008) for experiments involving humans. All subjects provided informed signed consent before participating in the study. A power analysis based on previously published data ${ }^{16}$ determined that a total of 12 subjects per group (test, control) is required to provide $95 \%$ power at $5 \%$ significance. A total of 18 prospective subjects (11males, 7 females) with an average age of 21.2(1.2) years were randomly recruited from. To qualify for the study, prospective subjects were required to be free of any clinical conditions that could affect normal somatosensory processing. Participants were required to complete a health history questionnaire to screen for the presence of any current musculoskeletal disorders or pain, history of spinal trauma (whiplash, fracture) and/or surgery, chronic or degenerative spine/joint disease and neurological conditions (neuropathy, numbness, weakness, special senses). Each of the 18 prospective participants qualified for the study and no subject withdrew from the study.

Employing a randomized crossover design, a research assistant assigned each qualified subject to their first intervention by blindly drawing labelled slips of paper from a bin. Subjects were blinded to group. Subjects were then scheduled to return for retesting with the opposite intervention after a minimum two- week washout interval. This study involved comparing skin temperature changes over predefined upper (Q2) and lower back (Q3) "target” regions (Figure 1) before and after the experimental induction of central sensitization within the C3-C5 spinal segments. Skin temperature was quantified using infrared thermography and recorded using 14-bit resolution with a portable infrared camera (ThermaCamTM SC2000; FLIR Systems Inc., Wilsonville, OR, USA) equipped with a built-in lens and calibrated with an emissivity value of 0.98 . Infrared images (thermographs) were obtained by a blinded, trained operator under controlled lighting conditions and from a constant distance of $1 \mathrm{~m}$ from the subject. We recorded separate images of upper and lower back skin regions to provide better spatial resolution of temperature.

Each subject acclimatized to room temperature for 15 minutes prior to receiving baseline thermographs. Topical cream (test, placebo) was then applied by a research assistant to the Q1 'treatment region' in both test and control groups to target the $\mathrm{C} 3-\mathrm{C} 5$ spinal segments. The Q1 region (C3-C5) was anatomically defined by the right scapular spine inferiorly, the vertebral spinous processes medially, right acromioclavicular joint laterally and the right clavicle and posterior border of the right sternocleidomastoid muscle anteriorly. ${ }^{17}$ The Q2 region (C3-C5) was anatomically distinguished by the left scapular spine inferiorly, cervical vertebral spinous processes medially, left acromioclavicular joint laterally and upper ridge of left trapezius muscle superiorly. ${ }^{17}$ The Q3 region (T10-L5) was anatomically defined by the tenth rib line ${ }^{18}$ superiorly, lumbar vertebral spinous processes medially, border of the trunk laterally and transverse line through bilateral posterior superior iliac spine inferiorly. ${ }^{19}$

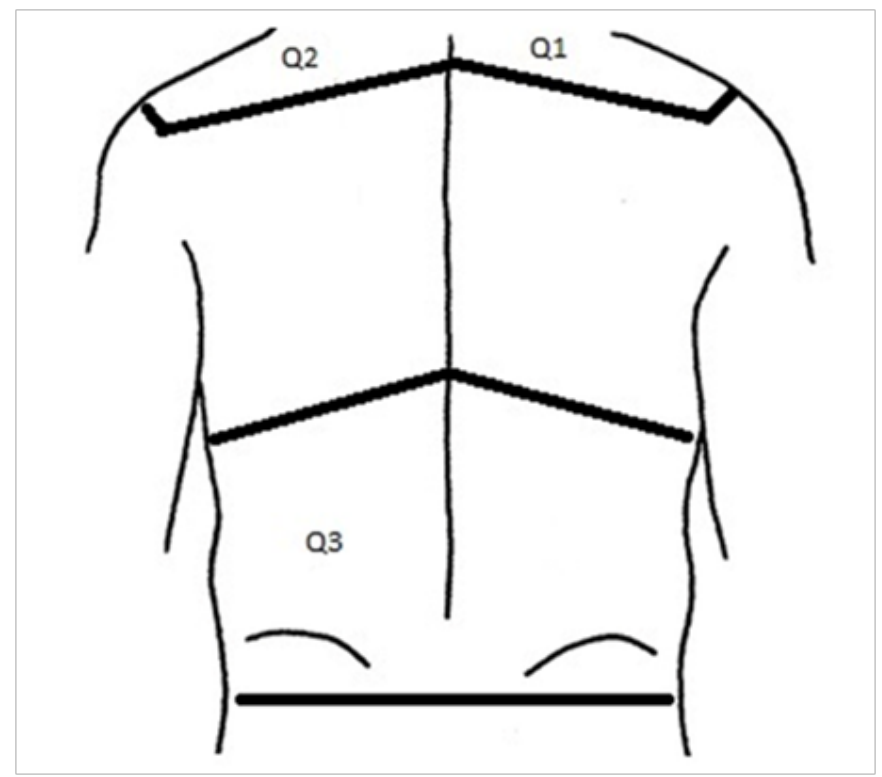

Figure I Upper and Lower Dermatomal Regions. Upper and Lower Regions are labelled QI-Q3 respectively. Upper Regions (QI, Q2) are anatomically defined by inferiorly by the scapular spine, laterally by the acromioclavicular joint, medially by the vertebral spinous process and anteriorly by the clavicular and sternocleidomastoid borders. The Lower Region (Q3) are anatomically defined superiorly by the tenth rib line, lumbar vertebral spinous process medially, lateral border of the trunk laterally and inferiorly by a transverse drawn through bilateral posterior superior iliac spines (PSIS). QI is the "treatment" region receiving the topical application (capsaicin, placebo) while Q2 and Q3 are the regions assessed for skin temperature change.

Subjects were then instructed that they were to receive a topical cream application by a research assistant. Test interventions received a topical application of $0.075 \%$ Zostrix (Hi Tech Pharmacal Co, Amityville, NY) to Q1 to evoke sensitization within the targeted C3C5 segment ${ }^{19}$. A thin layer of capsaicin covering Q1 was applied and massaged into the skin until visibly absorbed. Thermographs of Q2 and Q3 regions were then recorded at 10 (T10),20(T20) and 30(T30) minutes post-application. Control interventions received an application to Q1 of an inert, non-sensitizing placebo cream (Biotone, San Diego, CA). The identical application protocol was employed by the same research assistant for both test and control conditions.

In order to calculate skin temperatures from the defined regions of the back (Q2, Q3), as well as providing us with batch processing capability for the large number of image files collected in this study, we developed a custom software program in LabVIEW 8.5.1 (Austin, TX, USA). Native thermographs were recorded in 14-bit colour format and were converted to 8-bit grey scale images for further analysis (Photostudio 2000, ArcSoft Inc. Freemont, Calif. and USA). A custom $2^{\text {nd }}$ order polynomial calibration relating grey scale values to temperature was developed using a series of 31 thermography images of water at controlled temperature water baths between 24.6 and 38.0 degrees Celsius. The correlation between the predicted and actual temperatures was 0.999 . The precise regions of interest (Q2, Q3) were then traced in the 8-bit greyscale image using the freehand select 
tool in Photostudio (ArcSoft, V6). A mask was then created for each image in order to isolate the traced Q2 and Q3 regions. The mask was produced by blacking out all areas in the image outside of the traced Q2 and Q3 regions by assigning them a grey value of zero. Once the areas were masked, we computed the average temperature in Q2 and Q3 by calculating the arithmetic mean of the pixels within both regions using the custom Lab VIEW software. Representative images illustrating the steps in this process are provided in Figure 2.
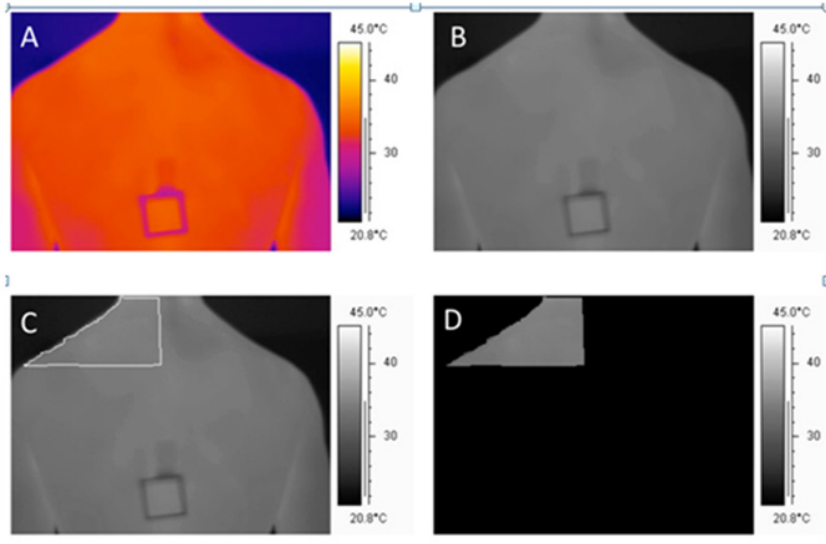

Figure 2 Thermographic image processing steps. Raw thermographic images were taken in 14-bit colour resolution with a portable infrared camera camera (ThermaCamTM SC2000; FLIR Systems Inc.,Wilsonville, OR, USA). (2A) It is a representative 14-bit colour image of the upper back taken at 20 minutes after applying capsaicin to the right neck and shoulder region. The I-inch square seen in the image is used for size calibration. (2B) The I4-bit colour image was converted to 8-bit greyscale image. (2C) The region of interest in the left shoulder and neck (Q2) was traced. Figure 2D) A mask was created by blacking out the areas outside the Q2 region to isolate the Q2 region for follow up analysis.

We collected a total of 31 trials, 18 test and 13 controls. One test trial and one control trial were disqualified due to technical software issues with the infrared camera. In addition, given that the aim of this study was to investigate the temporal relationship between experimentally induced central sensitization and autonomic response, a further two test trials were disqualified due to the lack of sensitization response to the topical capsaicin. Therefore, a total of 15 test trials ( 8 males, 7 females; age of 21.2(1.3) years) and 12 controls (5 males, 7 females; age of 21.2(1.3) years) were included in the analysis. We employed the brush allodynia protocol to assess for the experimental induction of central sensitization in participants. The brush allodynia protocol involves lightly brushing the skin with a fine filament brush to assess for changes in mechanosensation. In our protocol, we tested for changes in mechanical sensitivity of the skin region adjacent to $\mathrm{Q} 1$. We began by brushing the unaffected skin over the right paraspinal muscles distally at the L4-L5 spinal level (superior iliac crest) and progressed cephalad until the subject indicated a change in perception of the mechanical stimulus. We defined the change in perception as the point where the subject experienced the onset of enhanced mechanosensation, tingling or even mild discomfort in some cases. This point was marked with a non-toxic skin marker and the distance between this point and the nearest inferior margin of Q1 was measured and recorded $(\mathrm{mm})$, representing the Allodynia Score(16). Brush allodynia testing was performed at baseline (pre-capsaicin), 10, 20 and 30 minutes post-capsaicin.

\section{Statistical analysis}

The primary objective of this study was to compare differences in skin temperature change between the two target regions (Q2, Q3) under each treatment condition. We first calculated the average raw temperature change from baseline at each time interval for test versus control conditions. A 3-way ANOVA was then performed with time, group and region as the independent variables and average raw temperature change from baseline as the dependent variable. Significant results were followed up with post-hoc comparisons (Tukey's Test).

To assess for the presence of segmental temperature responses, we compared differences in raw temperature changes from baseline within Q2 and Q3 regions (Net Temperature Change) at each time interval under each treatment condition. The Net Temperature Change was calculated by subtracting the raw temperature change (from baseline) in Q3 from Q2, under both intervention conditions at each time interval. We then performed a 2-way ANOVA with Net Temperature Change as the dependent variable and time and group as the independent variables. Significant differences were followed up with post-hoc comparisons (Tukey's Test).

To validate the presence of secondary hyperalgesia, we tested the null hypothesis that the Allodynia Score under both intervention conditions was not different from zero. In order to assess differences between intervention groups, we also assessed whether there was a significant difference in Allodynia Score between treatment conditions. Average raw Allodynia Scores were calculated for each condition at each time interval $(10,20$ and $30 \mathrm{~min})$ post- capsaicin for each group. Average raw Allodynia Scores were then normalized to the 10 minute Allodynia Score to account for inter-subject variability. A 2-way ANOVA was performed using the normalized Allodynia Score as the dependent variable and time and group as the independent variables. Significant differences were followed up with post-hoc comparisons. Significant increases in Allodynia score supports the presence of central sensitization. ${ }^{20}$ Data was tested for normality using the Anderson-Darling test. The statistical analyst was not blinded to the treatment group. Statistical analysis was performed using PASW Statistics Software (Version 17.0.2). Significance was set for all analyses at $5 \%$.

\section{Results}

The raw temperature differences are summarized in Table 1. Analysis of variance of raw temperature changes from baseline demonstrated significant effects of time $(\mathrm{p}=0.026)$ and group $(p=0.016)$. Subsequent ANOVA performed at each time interval using independent variables of group and region as well as dependent variable of raw temperature change from baseline showed significant differences at all time intervals including $10(\mathrm{p}=0.003), 20(\mathrm{p}=0.030)$ and $30(\mathrm{p}=0.037)$ minutes. Post-hoc comparisons at each time interval revealed significant decrease in the mean temperature change from baseline in the test Q2 region when compared to all other groups (control Q2, test Q3, control Q3) at all time intervals.

Comparisons of test Q2 with control Q2 regions demonstrated significant decreases at $10(\mathrm{p}<0.001), 20(\mathrm{p}<0.001)$ and $30(\mathrm{p}=0.001)$ minutes in the test vs control groups. Comparison of test Q2 versus test Q3 groups also demonstrated significant decreases in temperature in test Q2 $\mathrm{v}$ test Q3 at $10(\mathrm{p}<0.001), 20(\mathrm{p}=0.018)$ and $30(\mathrm{p}=0.003)$ 
minutes. Significant decreases in test Q2 mean temperature was also observed in comparisons of test Q2 versus control Q3 at 10 $(\mathrm{p}=0.025)$ and $20(p=0.042)$ minutes while temperature differences approached significance at 30 minutes $(\mathrm{p}=0.075)$. No significance in mean temperature change from baseline was observed when comparing the control Q2, control Q3 and test Q3 groups at any time interval $(\mathrm{p}>0.050)$.

Table I Average Raw Temperature $\left({ }^{\circ} \mathrm{C}\right)$ Change from Baseline (pre-capsaicin). Raw temperatures were recorded with infrared thermography from each target skin region (upper back Q2 and lower back Q3) at 0 (baseline pre-capsaicin), 10, 20 and 30 minutes post-capsaicin application. Temperatures from each time interval were subtracted from the baseline (pre-capsaicin) value to obtain a raw temperature difference from baseline. Data presented as mean and standard deviation

\begin{tabular}{lclll}
\hline \multicolumn{2}{l}{ Time (minutes) Postsensitization } & & & \\
\hline Group & 0 & 10 & 20 & 30 \\
\hline Test-Upper & $0(0)$ & $-1.006(0.675)$ & $-I .160(0.77 I)$ & $-I .25 I(0.808)$ \\
Test-Lower & $0(0)$ & $0.166(0.752)$ & $-0.38 I(0.92 I)$ & $-0.363(0.696)$ \\
Control-Upper & $0(0)$ & $0.065(0.444)$ & $-0.145(0.5 I 3)$ & $-0.282(0.620)$ \\
Control-Lower & $0(0)$ & $0.024(I .497)$ & $-0.200(I .507)$ & $-0.346(I .662)$ \\
\hline
\end{tabular}

Univariate ANOVA performed on the Net Temperature Change between Q2 and Q3 regions revealed a significant effect of group $(\mathrm{p}<0.001)$; no significant effects of time $(\mathrm{p}=0.154)$ and time*group $(\mathrm{p}=0.100)$ were noted. Individual follow up comparisons of groups at each time interval revealed significant changes in average raw temperature difference between test Q2 and test Q3 regions at 10 $(\mathrm{p}=0.008), 20(\mathrm{p}=0.039)$ and $30 \quad(\mathrm{p}=0.040)$ minutes (Figure 3$)$.

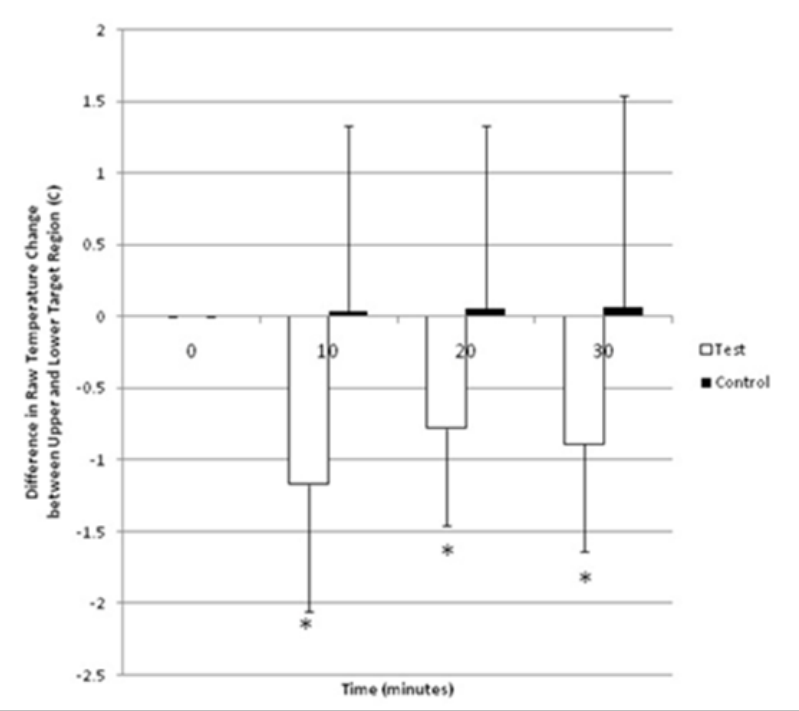

Figure 3 A Comparison of Raw Temperature Change from baseline between Upper (Q2) and Lower (Q3) Target Regions (Net Temperature Change). The C3-C5 spinal segments were sensitized in test subjects using topical capsaicin (Zostrix, 0.075\%; Hi-Tech Pharmacal, Amityville, NY, USA); controls received a non-sensitizing topical placebo (Biotone, San Diego, CA) application. Skin temperatures over the upper and lower target regions were recorded with infrared thermography at 0 (baseline), 10,20 and 30 minutes post application. Average raw temperature changes from baseline were calculated for upper (Q2) and lower (Q3) target regions in both test and controls. The difference in raw temperature change between upper and lower target regions was then calculated (Net Temperature Change) and these differences were compared at each time point in test subjects versus controls. Data is presented as mean and standard deviation. Significant differences are denoted by an asterisk $\left(^{*}\right)$.

Significant differences in normalized Allodynia Scores from zero were observed in test subjects at both $20(p<0.001)$ and $30(p<0.001)$ minutes while no difference was observed in controls at both $20(\mathrm{p}=0.313)$ and $30(\mathrm{p}=0.292)$ minutes. Analysis of variance of normalized Allodynia Scores demonstrated significant effects of time $(\mathrm{p}=0.001)$, group $(\mathrm{p}<0.001)$ and time* group $(\mathrm{p}=0.004)$. Post-hoc comparisons revealed significant increases of normalized Allodynia in test subjects at $20(\mathrm{p}<0.006)$ and $30(\mathrm{p}<0.001)$ minutes when compared with controls (Figure 4).

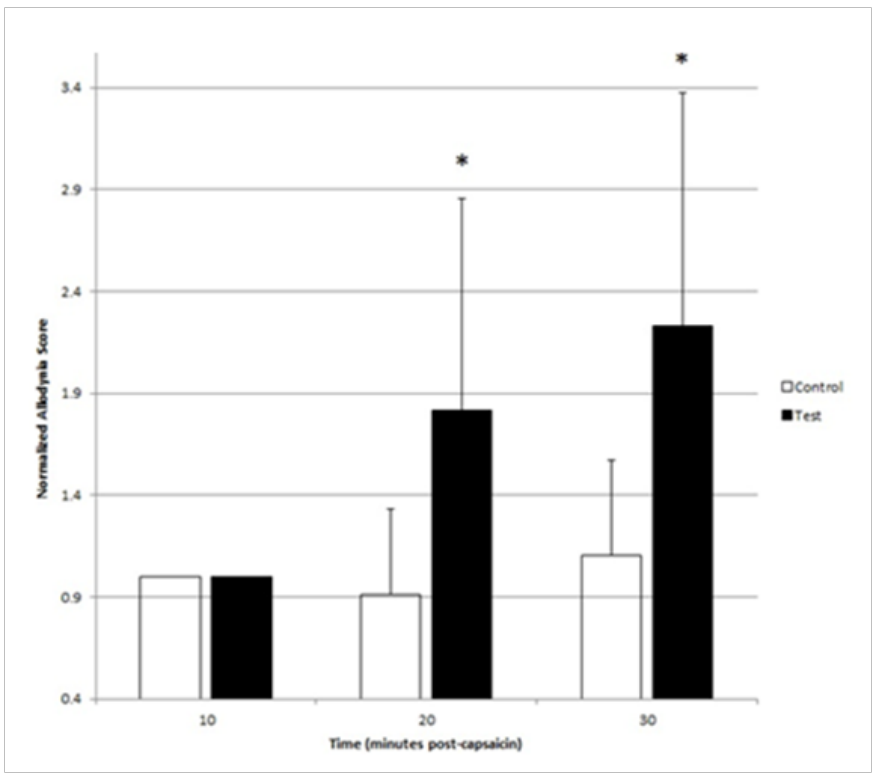

Figures 4 A Comparison of Allodynia Scores in Test versus Controls over Time. Average raw allodynia scores were calculated for each condition (test, control) at each time interval (10, 20, 30 minutes) post-capsaicin. Raw Allodynia Scores at each time interval were then normalized to 10 minute Allodynia Score. Data is presented as mean and standard deviation. Significant differences are denoted by an asterisk (*).

\section{Discussion}

Our hypothesis suggests that central sensitization may play an important causal physiologic mechanism mediating the pathophysiology and clinical manifestation of somatoautonomic responses in humans. Our study addressed the corollary to this by investigating the hypothesis that increasing central sensitization within a target spinal segment demonstrates a temporal association to changes in skin temperature expressed within neurosegmentally linked 
dermatomes in humans. Skin temperature is a common outcome used to experimentally quantify autonomic vasomotor control. ${ }^{21}$ Based on this rationale, we operationalized the somatoautonomic reflex on the basis of the temporal relationship between the experimental induction of central sensitization and skin temperature changes as quantified via infrared thermography.

The results of this study support our hypothesis by demonstrating that increasing central sensitization within the C3-C5 neural segments evoked significant decreases in average skin temperature within contralateral homologous dermatomes versus control (T10-L5) dermatomes. The average temperature of the upper back $(\mathrm{Q} 2)$ region was significantly reduced in test conditions at all time intervals post-sensitization when compared to all other groups (control Q2, test Q3, control Q3); no significant difference in average temperature was detected at any time interval between test Q3, control Q2 and control Q3 groups. Our results also demonstrate that the segmental mechanisms mediating the temperature changes were more predominant than supraspinal influences. The Net Temperature Change between Q2 and Q3 regions was significantly greater in test conditions, suggesting the presence of robust segmental temperature differences post-capsaicin, presumably due to somatoautonomic reflex mechanisms. In contrast, given that supraspinal mechanisms have the potential to impact the Q3 region under the test condition, the absence of significant temperature differences between test Q3 and control Q3 regions suggests that sensitization of the C3-C5 segments did not evoke meaningful supraspinal responses. Finally, our data reject the null hypothesis that Allodynia Scores are not different than zero in test subjects, but not in controls. Allodynia Scores were significantly higher in test subjects when compared with controls at 20 and 30 minutes post-capsaicin, confirming the induction of central sensitization within the test group, but not controls. A total of four subjects (three test, one control) were excluded from our data analysis. The primary aim of this study was to investigate the temporal relationship between experimentally evoked central sensitization within a target spinal segment and neurosegmentally arranged skin temperature changes in humans in vivo. We were unable to validate the induction of central sensitization in two subjects who failed to demonstrate an allodynia response post-capsaicin. In addition, one test and one control subject were excluded due to technical software issues with the infrared camera system. Allodynia is defined as increased sensitivity or pain to a non-painful (non-noxious) stimulus while hyperalgesia is the sensation of increased sensitivity or pain evoked by a normally painful (noxious) stimulus. Brush allodynia (mechanical hyperalgesia) testing is a commonly employed technique used to validate the presence of central sensitization. Although subjective, change in mechanosensation has been employed as a reliable clinical test for the presence of central sensitization. ${ }^{20}$ Using this technique, we were able to confirm the presence of allodynia within regions of secondary hyperalgesia surrounding the area of capsaicin application in all test subjects except for two; accordingly, we excluded these two subjects from our data analysis. None of the controls demonstrated allodynia post-capsaicin

Existing animal studies suggest that the nature of somatoautonomic responses can be variable. Somatoautonomic effects may exhibit both segmental and supraspinal mechanisms, the interactions of which are poorly understood. ${ }^{21}$ In general, the experimental literature reports that spinalized animals exhibit strong segmental somatoautonomic responses owing to the absence of descending supraspinal inhibitory influences, while CNS-intact animals appear to exhibit more generalized responses, however, segmental effects have also been reported. ${ }^{3}$

\section{Limitations}

The results of this study should be interpreted in light of several limitations. This was a seminal study aiming to investigate the temporal relationship between central sensitization and skin temperature change. Accordingly, we employed a young healthy population to limit age related confounders (such as chronic degenerative spine or joint disease) that could influence central sensitization, ${ }^{22}$ in order to study these mechanisms under normal, healthy conditions. Furthermore, we employed topical capsaicin to evoke central sensitization in this study, however, the intensity and quality of the somatoautonomic response may be dependent on several intrinsic factors of the stimulus, including the nature (noxious, innocuous), intensity and anatomic origin of the triggering noxious stimulus (superficial, deep).$^{23}$ Previous research has shown that deep tissue noxious pain (capsaicin injection) evokes a greater and longer lasting sensitization response compared with less invasive topical applications of capsaicin. ${ }^{24}$ Given that we observed significant skin temperature changes using a mild $(0.075 \%)$ topical dose of capsaicin, it is possible that higher doses of topical capsaicin or intradermal capsaicin injection could evoke significantly enhanced temperature responses. Although the possibility of subject group bias exists, the control cream we employed was an inert, non-sensitizing cream specifically chosen with similar properties (colour, texture) to the topical capsaicin cream. Furthermore, subjects were instructed that they would receive one of two topical interventions but were unable to identify their group allocation. Even if they correctly discerned their group allocation, participants would not be able to physiologically impact skin temperature in the segmental patterns observed in this study; therefore, we are confident that the potential for subject group bias was negligible in this study.

We employed infrared thermography to quantify skin temperature changes. Although infrared thermography demonstrates variability, ${ }^{25}$ the stability of paraspinal and back temperature measurements have previously been reported to be high, ${ }^{26,27}$ and the quantification of skin temperature using infrared thermography was reported to be as reliable as contact thermometry. ${ }^{28}$ Skin temperature (thermoregulation) is a function of cutaneous and subcutaneous blood flow which is regulated by the ANS. ${ }^{29}$ Despite the fact that variability in skin temperature can be significantly influenced by a number of factors including variation in raw baseline temperatures, blood flow (hemodynamics) and ambient temperature,30 our data demonstrates robust neurosegmental changes in skin temperature immediately post-capsaicin. These findings highlight a potentially important causal role for central sensitization in the physiologic manifestation of somatoautonomic responses in humans.

The observed segmental temperature responses post-capsaicin in this study have not been previously demonstrated experimentally in vivo in humans and the anatomic basis for this segmental response in the cervical spine is unclear. Sympathetic input to the head, upper limbs and cervical spine originates in the upper thoracic spine. ${ }^{17}$ Upper thoracic pre-ganglionic sympathetic fibers exit the intermediolateral column of the spinal cord and ascend within the sympathetic trunk to synapse with post-ganglionic fibers located in the cervical sympathetic ganglia. Animal models confirm the presence of a segmental arrangement between ascending pre-ganglionic and post-ganglionic fibers within the stellate ganglion. ${ }^{30}$ In addition, post-ganglionic fibers 
also receive synaptic inputs from axon collaterals of sensory afferents located within the dorsal root ganglia. ${ }^{31}$ These observations suggest that the cervical sympathetic ganglia may act to segmentally integrate and coordinate both central and peripheral somatosensory inputs with visceral efferent pathways. ${ }^{31}$

\section{Future directions}

This study is the first to demonstrate a temporal relationship between the experimental induction of central sensitization and neurosegmentally related skin temperature changes in humans. The findings of this study draw attention to several important directions for future research. While the observed temporal response is an important initial finding, it does not confirm causality. Future research should advance this question by investigating post-ganglionic autonomic responses using direct nerve recordings in humans (microneurography). Furthermore, elucidating the dose-response relationship between central sensitization and skin temperature change in humans would provide further insight into the causal relationship between central sensitization and somatoautonomic responses in humans. The anatomic and physiologic mechanisms by which the cervical sympathetic ganglia segmentally integrate pre-ganglionic afferent and visceral efferent pathways should be established using animal models to enable direct access and measurement of these pathways. Finally, while the external validity of the observations in this study are limited given the young healthy cohort, future research should study these responses in older and clinical populations to better understand the potential role of age and other comorbidities on these mechanisms.

\section{Conclusion}

This study is the first to demonstrate a temporal relationship between the experimental induction of central sensitization and neurosegmental skin temperature change in humans. These findings provide additional support the hypothesis that central sensitization may play a causal role in the clinical manifestation of somatoautonomic responses in humans. These findings have potentially significant implications to the conservative therapeutic management of somatoautonomic disorders. Previous research has shown that manual therapy, such as spinal manipulative therapy (SMT), modulates autonomic expression and visceral hypersensitivity, ${ }^{32}$ and recent research suggests that it may do so by specifically modulating central sensitization. ${ }^{33}$ Improving our understanding of these mechanisms will contribute to our understanding of how conservative manual therapies may contribute and advance the conservative management of autonomic dysfunction and/or visceral hypersensitivity in humans.

\section{Acknowledgements}

None.

\section{Conflict of interest}

The author declares no conflict of interest.

\section{References}

1. Benarroch EE. Pain-autonomic interactions. Neurol Sci. 2006;27(2):130133.

2. Sato A, Schmidt RF. The modulation of visceral functions by somatic afferent activity. Jpn J Physiol. 1987;37(1):1-17.

3. Sato A. Neural mechanisms of autonomic responses elicited by somatic sensory stimulation. Neurosci Behav Physiol. 1997;27(5):610-621.
4. Delaney JP, Leong KS, Watkins A, et al. The short-term effects of myofascial trigger point massage therapy on cardiac autonomic tone in healthy subjects. J Adv Nurs. 2002;37(4):364-371.

5. Budgell BS. Reflex effects of subluxation: the autonomic nervous system. J Manipulative Physiol Ther. 2000;23(2):104-106.

6. Burton AR, Birznieks I, Spaak J, et al. Effects of deep and superficial experimentally induced acute pain on skin sympathetic nerve activity in human subjects. Experimental Brain Research. 2009;195(2):317-324.

7. Burton AR, Birznieks I, Bolton PS, et al. Effects of deep and superficial experimentally induced acute pain on muscle sympathetic nerve activity in human subjects. J Physiol. 2009;587(1):183-193.

8. Cameron DM, Brennan TJ, Gebhart GF. Hind paw incision in the rat produces long-lasting colon hypersensitivity. J Pain. 2008;9(3):246-253.

9. Sato A, Sato Y, Schmidt RF. Changes in blood pressure and heart rate induced by movements of normal and inflamed knee joints. Neuroscience Letters. 1984;52(1-2):55-60.

10. Kaufman A, Sato A, Sato Y, et al. Reflex changes in heart rate after mechanical and thermal stimulation of the skin at various segmental levels in cats. Neuroscience. 1997;2(1):103-109.

11. Sato A, Sato Y, Sugimoto H, et al. Reflex changes in the urinary bladder after mechanical and thermal stimulation of the skin at various segmental levels in cats. Neuroscience. 1977;2(1):111-117.

12. Takahashi Y, Hirayama J, Nakajima Y. Segmental regulation pattern of body surface temperature in the rat hindlimb. Brain Res. 2002;947(1):100-109.

13. Calvino B. [Neural basis of pain]. Psychol Neuropsychiatr Vieil. 2006;4(1):7-20.

14. Cervero F. Visceral pain-central sensitisation. Gut. 2000;47(4):56-57.

15. Sarkar S, Hobson AR, Furlong PL, et al. Central neural mechanisms mediating human visceral hypersensitivity. Am J Physiol Gastrointest Liver Physiol. 2001;281(5):1196-1202.

16. Srbely JZ, Dickey JP, Bent LR, et al. (2010) Capsaicin-induced central sensitization evokes segmental increases in trigger point sensitivity in humans. J Pain. 2010;11(7):636-643.

17. Moore KL. Clinically Oriented Anatomy. 2nd ed, Baltimore: Williams \& Wilkins; 1985. p.1-1101.

18. Jung $\mathrm{CW}$, Bahk JH, Lee JH, et al. The tenth rib line as a new landmark of the lumbar vertebral level during spinal block. Anaesthesia. 2004;59(4):359-63.

19. Dirks J, Petersen KL, Dahl JB. The heat/capsaicin sensitization model: a methodologic study. J Pain. 2003;4(3):122-128.

20. Ashkenazi A, Young WB. Dynamic mechanical (brush) allodynia in cluster headache. Headache. 2004;44(10):1010-1012.

21. Uematsu S, Edwin DH, Jankel WR, et al. Quantification of thermal asymmetry. Part 1: Normal values and reproducibility. J Neurosurg. 1998;69(4):552-555.

22. Kimura A, Ohsawa H, Sato A, et al. Somatocardiovascular reflexes in anesthetized rats with the central nervous system intact or acutely spinalized at the cervical level. Neuroscience Research. 1995;22(3):297305.

23. Schaible HG, Ebersberger A, von Banchet GS. Mechanisms of pain in arthritis. 2002;966:343-354.

24. Sluka KA. Stimulation of deep somatic tissue with capsaicin produces long-lasting mechanical allodynia and heat hypoalgesia that depends on early activation of the cAMP pathway. $J$ Neurosci. 2002;22(13):56875693. 
25. Zaproudina N, Varmavuo V, Airaksinen O, et al. Reproducibility of infrared thermography measurements in healthy individuals. Physiol Meas. 2008;29(4):515-524.

26. Roy R, Boucher JP, Comtois AS. Validity of infrared thermal measurements of segmental paraspinal skin surface temperature. $J$ Manipulative Physiol Ther. 2006;29(2):150-155.

27. Owens EF, Hart JF, Donofrio JJ, et al. Paraspinal skin temperature patterns: an interexaminer and intraexaminer reliability study. $J$ Manipulative Physiol Ther. 2004;27(3):155-159.

28. van den Heuvel CJ, Ferguson SA, Dawson D, et al. Comparison of digital infrared thermal imaging (DITI) with contact thermometry: pilot data from a sleep research laboratory. Physiol Meas. 2003;24(3):717-725.

29. Jung AZ. Clinical applications of thermographic methods. In: Jung AZ, editor. Thermographic methods in medical diagnostics. Poland: Medpress; 1998. p. 37-59.
30. Asamoto K. Network of the sympathetic nervous system: focus on the input and output of the cervical sympathetic ganglion. Anat Sci Int. 2005;80(3):132-140.

31. Kummer W, Oberst P. Neuronal projections to the guinea pig stellate ganglion investigated by retrograde tracing. J Auton Nerv Syst. 1993;42(1):71-80

32. Bolton PS, Budgell B. Visceral responses to spinal manipulation. $J$ Electromyogr Kinesiol. 2012;22(5):777-784.

33. Srbely JZ, Vernon H, Lee D, et al. Immediate effects of spinal manipulative therapy on regional antinociceptive effects in myofascial tissues in healthy young adults. J Manipulative Physiol Ther. 2013;36(6):333-341. 